Vol. 65 (2002) [461-466]

\title{
SOME REMARKS ON CS MODULES AND SI RINGS
}

\section{Dinh VAN HUYNh}

\begin{abstract}
We discuss some results on CS modules, SI rings, and SC rings. Then we consider the question of when, over a right SC ring $R$, every right $R$-module is CS. In Theorem 3.1 we show that this is the case if and only if $R$ is a right countably $\Sigma$-CS ring. In light of this, we give an example showing that a result proved by Chen (2000) is incorrect. Furthermore, Theorem 4.1 shows that the assumptions of Chen (2000) can be weakened considerably.
\end{abstract}

\section{CS Modules and CS Rings}

We consider associative rings with identity and all modules are unitary modules. For a module $M$ over a ring $R$ we write $M_{R}$ to indicate that $M$ is a right $R$-module. The socle and the Jacobson radical of $M$ are denoted by Soc $(M)$ and $J(M)$, respectively. If $M$ has finite composition length, then its length is denoted by $l(M)$.

A module $M$ is called a CS module if every submodule of $M$ is essential in a direct summand of $M$. A ring $R$ is said to be a right CS ring if $R_{R}$ is CS. Moreover, a module $M$ is defined to be a (countably) $\Sigma$-CS module if $M^{(A)}$ (respectively, $M^{(\mathbb{N})}$ ) is $\mathrm{CS}$ for any set $A$. Note that $\mathbb{N}$ denotes the set of all positive integers. A ring $R$ is right (countably) $\Sigma$-CS if $R_{R}$ is (countably) $\Sigma$-CS. If every right $R$-module is CS, then $R$ is defined to be right CS-semisimple. The structure of right CS-semisimple rings was obtained by Dung-Smith [4] as follows.

THEOREM 1.1. ([4]) For a ring $R$ the following conditions are equivalent:

(a) $R$ is right CS-semisimple.

(b) $R$ is right and left Artinian, right and left serial with $J(R)^{2}=0$.

(c) $R_{R}=\bigoplus_{i=1}^{n} R_{i}$ where each $R_{i}$ is either simple or uniform of length 2 and injective.

(d) The left-handed version of (a) and (c).

From Theorem 1, right CS-semisimple rings are left CS-semisimple. Hence we simply call these rings CS-semisimple rings. There are CS-semisimple rings which are not quasi-Frobenius (QF) (see Remark 3.4).

Right $\Sigma$-CS rings were first studied by Oshiro [10] under the name "right co-H rings". The structure of these rings was obtained by Oshiro in the following theorem.

Received 25th October, 2001

Copyright Clearance Centre, Inc. Serial-fee code: 0004-9727/02 \$A2.00+0.00. 
ThEOREM 1.2. ([10, 11], see also [12].) Every right $\Sigma$-CS ring $R$ is right and left Artinian, and the injective hull $E\left(R_{R}\right)$ is projective.

By Theorem 1.2 , if $R$ is a right $\Sigma$-CS ring then $R_{R}=R_{1} \oplus \cdots \oplus R_{n}$ where each $R_{i}$ is a local Artinian module. Since $E\left(R_{R}\right)$ is projective, it is finitely generated, and moreover, $E\left(R_{R}\right)=\bigoplus_{i=1}^{n} E_{i}$ where each $E_{i}$ is isomorphic to some $R_{j} \in\left\{R_{1}, \ldots, R_{n}\right\}$ (see $[1,27.11])$. These facts are very useful in understanding the structure of right $\Sigma$-CS rings.

Notice that $\Sigma$-injective modules were studied first by Faith (see, for example, [6, pp. 111-114]) who proved that a ring $R$ is QF if and only if $R$ is right countably $\sum$-injective.

From Theorem 1.2 and the main result of [8], the following statement holds.

Proposition 1.3. A right Noetherian, right countably $\Sigma$-CS ring is right $\Sigma-C S$, and right and left Artinian.

More generally, J. Clark and R. Wisbauer [3] proved that a right countably $\Sigma$-CS ring with acc on right annihilators is right $\Sigma$-CS (see also $[5,11.12]$ ). But here we restrict ourselves to the Noetherian case.

Rings, over which every CS right $R$-module is $\Sigma$-CS, were studied in [9], where the following theorem was obtained.

THEOREM 1.4. ([9]) If $R$ is a ring such that every CS right $R$-module is $\Sigma$-CS, then $R$ is right Artinian.

When handling finitely generated CS modules the following simple fact is very useful. It implies for example, a right CS semiperfect ring is a direct sum of (finitely many) uniform right ideals.

Lemma 1.5. (See [5, 9.1].) Let $M$ be a finitely generated CS module. If $M$ contains an infinite direct sum of nonzero submodules $N=\bigoplus_{i=1}^{\infty} N_{i}$, then the factor module $M / N$ does not have finite uniform dimension.

Osofsky and Smith [13] proved a strong theorem forcing certain cyclic (or even finitely generated) modules to have finite uniform dimension.

THEOREM 1.6. ([13], see also $[5,7.13]$.) If $M$ is a cyclic module such that every cyclic submodule of any factor module of $M$ is CS, then $M$ is a direct sum of (finitely many) uniform modules.

For more information on CS modules we refer to the book [5].

\section{Structure of SI Rings}

A ring $R$ is called a right SI ring if every singular right $R$-module is injective. Left 
SI rings are defined similarly. SI rings were introduced and investigated by Goodearl [7], where the structure of right SI rings was obtained.

TheOREM 2.1. ([7,3.11].) A ring $R$ is right $S I$ if and only if $R$ is right nonsingular and $R$ has a ring-direct decomposition $R=K \oplus R_{1} \oplus \cdots \oplus R_{n}$ where $K / \operatorname{Soc}\left(K_{K}\right)$ is semisimple, and each $R_{i}$ is Morita-equivalent to a right SI domain that is not a division ring.

Note that a right SI domain $D$ is right Noetherian and right hereditary. Moreover, for any nonzero right ideal $C \subseteq D, D / C$ is a semisimple right $D$-module. From Theorem 2.1, if $R$ is a right SI ring, then $R / \operatorname{Soc}\left(R_{R}\right)$ is right Noetherian. Hence by Lemma 1.5, if $R$ is moreover a right CS ring then $R$ is right Noetherian. Notice further, that using Theorem 1.6, it can be shown that a ring $R$ is right SI if and only if every cyclic singular right $R$-module is injective (see [13]).

Motivated by the concept of SI rings, Rizvi and Yousif [14] defined right (left) SC rings. A ring $R$ is called a right (left) $\mathrm{SC}$ ring if every (finitely generated) singular right (left) $R$-module is continuous. By [14, Corollary 3.3], if $R$ is a right SC ring then $J(R) \subseteq \operatorname{Soc}\left(R_{R}\right)$, and $R / \operatorname{Soc}\left(R_{R}\right)$ is a right Noetherian, right $\mathrm{V}$ and right SI ring. From this, Lemma 1.5 and Theorem 2.1, the following holds obviously.

Propos I I ION 2.2. If $R$ is a right $C S$, right $S C$ ring, then $R$ is a right Noetherian ring such that $R / \operatorname{Soc}\left(R_{R}\right)=A \oplus B$ where $A$ is a semisimple Artinian ring and $B$ is a right Noetherian right $S I$ ring with zero right socle.

\section{When ARE SC Rings CS-Semisimple?}

The following theorem characterises all right $\Sigma$-CS, right SC rings.

ThEOREM 3.1. For a right $S C$ ring $R$, the following conditions are equivalent:

(a) $R$ is a right countably $\Sigma$-CS ring.

(b) Every right (and left) $R$-module is $C S$.

Proof: $(a) \Rightarrow(b)$. By Proposition 2.2, $R$ is a right Noetherian ring. Hence by Proposition 1.3, $R$ is right (and left) Artinian right $\Sigma$-CS. Then $R_{R}=R_{1} \oplus \cdots \oplus R_{n}$, where each $R_{i}$ is a uniform local right $R$-module. Moreover, since $R / \operatorname{Soc}\left(R_{R}\right)$ is semisimple (see Proposition 2.2), each $R_{i} / \operatorname{Soc}\left(R_{i}\right)$ is either zero or simple, or equivalently, $l\left(R_{i}\right)$ is 1 or 2 . Hence, if $l\left(R_{i}\right)=2$, then $R_{i}$ is injective (see the discussion following Theorem 1.2). Therefore, by Theorem 1.1, every right (and left) $R$-module is CS, proving (b). The implication $(b) \Rightarrow(a)$ is trivial.

COROLlaRY 3.2. Let $R$ be a right quasi-continuous, right SC ring. If $R_{R}^{(\mathrm{N})}$ is $C S$, then $R$ is a $Q F$ ring.

Proof: By Theorem 3.1, $R$ is CS-semisimple. Hence $R_{R}=R_{1} \oplus \cdots \oplus R_{n}$ where 
each $R_{i}$ is either simple or uniform injective and of length 2 . Since $R$ is right quasicontinuous, each $R_{i}$ is $R_{j}$-injective for $i \neq j$. As $R$ is right $\Sigma$-CS, $E\left(R_{i}\right)$ is isomorphic to some $R_{k} \in\left\{R_{1}, \ldots, R_{n}\right\}$ (see the discussion following Theorem 1.2). In particular, $R_{i}$ embeds in $R_{k}$. If $R_{i} \not R_{k}$, then $R_{k}$ contains a submodule $U$ with $U \cong R_{i}$. But as $R_{i}$ is $R_{k}$-injective, $U$ splits in $R_{k}$, a contradiction. Hence $R_{i} \cong R_{k}$, proving that $R_{i}$ is injective. Thus $R$ is right self-injective. This shows that $R$ is QF.

REMARK 3.3. Corollary 3.2 shows that in [2, Theorem 2] instead of "every CS right $R$-module is $\Sigma$-CS" it is enough to assume that $R$ is right countably $\Sigma$-CS.

REMARK 3.4. The matrix ring of the form $R=\left[\begin{array}{cc}\mathbb{R} & \mathbb{R} \\ 0 & \mathbb{R}\end{array}\right]$, where $\mathbb{R}$ is the field of real numbers, is clearly a ring of Theorem 3.1. But this ring is not right self-injective, since $E\left(R_{R}\right)=\left[\begin{array}{ll}\mathbb{R} & \mathbb{R} \\ \mathbb{R} & \mathbb{R}\end{array}\right]$. Hence $R$ can not be a $\mathrm{QF}$ ring. This example and Theorem 3.1 show that Theorem 4 in [2] is incorrect. Notice further, in the proof of [2, Theorem 4], the author of [2] did not distinguish between a direct sum of rings and a direct sum of modules, while these are quite different when applied on a ring. For example, the ring $R$ in our example, is a direct sum of two right ideals $\left(\left[\begin{array}{cc}\mathbb{R} & \mathbb{R} \\ 0 & \mathbb{R}\end{array}\right]=\left[\begin{array}{ll}\mathbb{R} & \mathbb{R} \\ 0 & 0\end{array}\right] \oplus\left[\begin{array}{ll}0 & 0 \\ 0 & \mathbb{R}\end{array}\right]\right)$, but as a ring, $\left[\begin{array}{ll}\mathbb{R} & \mathbb{R} \\ 0 & \mathbb{R}\end{array}\right]$ is indecomposable.

\section{Rings with Condition (*)}

In [2] the condition $\left(^{*}\right)$ for a ring $R$ was defined as follows: For any pair of orthogonal primitive idempotents $f, g \in R, f R g=0$ if and only if $g R f=0$.

THEOREM 4.1. Let $R$ be a left perfect right nonsingular ring. Then $R$ satisfies $\left(^{*}\right)$ if and only if $R$ is semisimple Artinian.

PROOF: It is easy to see that every semisimple Artinian ring satisfies $\left(^{*}\right)$. Now let $R$ be a left perfect ring. By $[\mathbf{1}, 28.4], R$ has an essential right socle. Assume further that $R$ is right nonsingular, and let $S$ be a minimal right ideal of $R$. Then $S=x R$ for some $x \in S$. It follows that $S \cong R / \operatorname{ann}_{R}(x)$ where $\operatorname{ann}_{R}(x)=\{y \mid y \in R, x y=0\}$. Since $R$ is right nonsingular, $S_{R}$ is projective. Therefore, $R_{R}=T \oplus \operatorname{ann}_{R}(x)$ for some right ideal $T \subseteq R$ with $T \cong S$. This means:

(1) For every left perfect right nonsingular ring $R, R_{R}=T \oplus R^{\prime}$ where $T$ is a minimal right ideal of $R$.

Since $R$ is left perfect, $R_{R}=R_{1} \oplus \cdots \oplus R_{t} \oplus R_{t+1} \oplus \cdots \oplus R_{n}$, where each $R_{i}$ is an indecomposable right ideal of $R$. Moreover, we may assume that $R_{1}, \ldots, R_{t}$ are minimal right ideals, and $R_{t+1}, \ldots, R_{n}$ are not minimal. We may assume also that each $R_{i}, 1 \leq i \leq t$, is generated by an idempotent $e_{i}$, that is, $R_{i}=e_{i} R$, and 
each $R_{j}, t+1 \leq j \leq n$, is generated by an idempotent $f_{j}$, that is, $R_{j}=f_{j} R$. Furthermore, we can choose the $e_{i}{ }^{\prime} s, f_{j}{ }^{\prime} s$ such that the set $\left\{e_{1}, \ldots, e_{t}, f_{t+1}, \ldots, f_{n}\right\}$ is orthogonal. If there is $0 \neq \varphi \in \operatorname{Hom}_{R}\left(f_{j} R, e_{i} R\right)$, then $\varphi\left(f_{j} R\right)=e_{i} R$. Hence $\operatorname{Ker} \varphi$ splits in $f_{j} R$. As $f_{j} R$ is a local module, this implies that $\operatorname{Ker} \varphi=0$, but this is impossible because $l\left(f_{j} R\right)>l\left(e_{i} R\right)=1$. Hence $\operatorname{Hom}_{R}\left(f_{j} R, e_{i} R\right)=0$. Now let $R$ satisfy condition $\left(^{*}\right)$. Then $\operatorname{Hom}_{R}\left(f_{j} R, e_{i} R\right)=e_{i} R f_{j}=0,(1 \leq i \leq t, t+1 \leq j \leq n)$, implies $0=f_{j} R e_{i}=\operatorname{Hom}_{R}\left(e_{i} R, f_{j} R\right),(1 \leq i \leq t, t+1 \leq j \leq n)$. Hence we get a ring-direct sum $R=A \oplus B$ where $A_{A}=R_{1} \oplus \cdots \oplus R_{t}$, and $B_{B}=R_{t+1} \oplus \cdots \oplus R_{n}$. Therefore, $A$ is a semisimple Artinian ring, and $B$ is a right nonsingular left perfect ring such that $B_{B}$ is a direct sum of local submodules of length $>1$. Suppose $B \neq 0$. By the Krull-Schmidt Theorem (see $[1,12.9]$ ), $B$ does not contain a minimal right ideal as a direct summand of $B_{B}$. This is a contradiction to (1). Hence $B=0$, and so, $R$ is semisimple Artinian, as desired.

COROLlaRY 4.2. Let $R$ be a right nonsingular ring such that every CS right $R$-module is $\Sigma$-CS. Then $R$ satisfies $\left(^{*}\right)$ if and only if $R$ is semisimple Artinian.

Proof: By Theorem 1.4, $R$ is right Artinian. Hence the statement follows from Theorem 4.1.

REMARK 4.3. In [2, Theorem 3] it was shown that, if $R$ is a right SI right CS ring with $\left({ }^{*}\right)$ such that every CS right $R$-module is $\Sigma$-CS, then $R$ is QF. In light of Theorems 4.1 and 2.1, the right SI right CS assumptions in this statement can be replaced by the much weaker assumption of right nonsigularity; and secondly, it should be concluded that $R$ is semisimple Artinian. In fact, a right nonsingular QF ring is semisimple Artinian.

If we remove the condition "every CS right $R$-module is $\Sigma$-CS", we still get the structure of $R$ as follows.

COROLlaRY 4.4. Let $R$ be a right SI right CS ring. Then $R$ satisfies condition $\left({ }^{*}\right)$ if and only if $R$ is a ring-direct sum of a semisimple Artinian ring and a right SI ring with zero right socle.

Proof: By Lemma 1.5 and Theorem 2.1, a right SI right CS ring $R$ is a direct sum of a right Artinian ring $A$ and a right SI ring $B$ with $\operatorname{Soc}(B)=0$. (Note that every right SI ring is right nonsingular (see Theorem 2.1).) If $R$ satisfies $\left(^{*}\right.$ ) then $A$ is semisimple Artinian by Theorem 4.1. Conversely, let $R=A \oplus B$, where $A$ is semisimple Artinian and $B$ is right SI right CS with zero right socle. By Theorem 2.1, $B$ is a direct sum of simple Noetherian right CS rings. Hence we may assume that $B$ is simple. Write $B=B_{1} \oplus \cdots \oplus B_{k}$ where each $B_{i}$ is uniform. As $B$ is a prime Noetherian ring, each $B_{i}$ is subisomorphic to $B_{j}$. Hence $\operatorname{Hom}\left(B_{i}, B_{j}\right) \neq 0$ for all $i, j$. This means, for any orthogonal primitive idempotents $f, g \in B$, we have $f B g \neq 0$. Thus $B$, and hence $R(=A \oplus B)$ satisfies $(*)$. 


\section{REFERENCES}

[1] F.W. Anderson and F.R. Fuller, Rings and categories of modules (2nd Edition), Graduate Texts in Mathematics 13 (Springer-Verlag, New York, 1992).

[2] M.S. Chen, 'On CS rings and QF rings', Southeast Asian Bull. Math. 24 (2000), 25-29.

[3] J. Clark and R. Wisbauer, ' $\Sigma$-extending modules', J. Pure Appl. Algebra 104 (1995), 19-32.

[4] N.V. Dung and P.F. Smith, 'Rings for which certain modules are CS', J. Pure Appl. Algebra 102 (1995), 257-265.

[5] N.V. Dung, D.V. Huynh, P.F. Smith and R. Wisbauer, Extending modules, Pitman Research Notes in Mathematics Series 313 (Longman Scientific and Technical, Harlow, 1994).

[6] C. Faith, Algebra II: Ring theory (Springer Verlag, Berlin, New York, 1976).

[7] K.R. Goodearl, Singular torsion and the splitting properties, Mem. Amer. Math. Soc. 124 (American Mathematical Society, Providence, R.I., 1972).

[8] D.V. Huynh, 'A right countably $\Sigma$-CS ring with acc or dcc on projective principal right ideals is left artinian and QF-3', Trans. Amer. Math. Soc. 347 (1995), 3131-3139.

[9] D.V. Huynh, S.K. Jain and S.R. López-Permouth, 'Rings characterized by direct sums of CS modules', Comm. Algebra 28 (2000), 4219-4222.

[10] K. Oshiro, 'Lifting modules, extending modules and their applications to QF rings', Hokkaido Math. J. 13 (1984), 310-338.

[11] K. Oshiro, 'On Harada rings I, II, III', Math. J. Okayama Univ. 31 (1989), 161-178, 179-188; 32 (1990), 111-118.

[12] K. Oshiro, 'Theories of Harada in artinian rings and applications to classical artinian rings', in International Symposium on Ring Theory, (G.F. Birkenmeier, J.K. Park, S. Park, Editors), Trends Math. (Birkhäuser, Boston, 2001), pp. 279-301.

[13] B.L. Osofsky and P.F. Smith, 'Cyclic modules whose quotients have all complement submodules direct summands', J. Algebra 139 (1991), 342-354.

[14] S.T. Rizvi and M.F. Yousif, 'On continuous and singular modules', in Non-commutative Ring Theory, (S.K. Jain and S.R. López-Permouth, Editors), Lecture Notes in Math. 1448 (Springer Verlag, Berlin, 1990), pp. 116-124.

\footnotetext{
Department of Mathematics

Ohio University

Athens $\mathrm{OH} 45701$

United States of America

e-mail: huynh@math.ohiou.edu
} 